\title{
String solutions in Chern-Simons-Higgs model coupled to an axion
}

\author{
J. López-Sarrión, ${ }^{1}$ E. F. Moreno, ${ }^{2,3, *}$ F. A. Schaposnik, ${ }^{2, \dagger}$ and D. Slobinsky ${ }^{2}$ \\ ${ }^{1}$ Departamento de Física, Universidad de Santiago de Chile, Casilla 307, Santiago, Chile \\ ${ }^{2}$ Departamento de Física, Facultad de Ciencias Exactas, Universidad Nacional de La Plata, C.C 67, 1900 La Plata, Argentina \\ ${ }^{3}$ Department of Physics, West Virginia University, Morgantown, West Virginia 26506-6315, USA
}

(Received 10 March 2006; published 8 June 2006)

\begin{abstract}
We study a $d=2+1$ dimensional Chern-Simons gauge theory coupled to a Higgs scalar and an axion field, finding the form of the potential that allows the existence of self-dual equations and the corresponding Bogomolny bound for the energy of static configurations. We show that the same conditions allow for the $N=2$ supersymmetric extension of the model, reobtaining the BPS equations from the supersymmetry requirement. Explicit electrically charged vortexlike solutions to these equations are presented.
\end{abstract}

DOI: 10.1103/PhysRevD.73.125007

PACS numbers: 11.27.+d, 11.10.Kk, 12.60.Jv

\section{INTRODUCTION}

There has been recently a revival in the interest on Abrikosov-Nielsen-Olesen flux tubes in connection with supergravity models and the realization of cosmic superstrings. Indeed, although such cosmic size superstrings were originally excluded in the context of perturbative string theory [1], it became clear recently that the question should be revisited as the relevance of branes and new kinds of extended objects was understood (see [2] and references therein). In order to understand the nature and structure of such stringy cosmic strings, the embedding of BPS objects in supersymmetry and supergravity models has become an active area of research so that the properties of BPS solitons, their connections with the supersymmetry algebra and their cosmological applications have been discussed by many authors [3-16].

Having in mind the study of BPS solitons in a string theory context, it is natural to consider models where an axion field is included. In particular, an $N=1$ globally supersymmetric model in $d=3+1$ dimensions consisting of an axion superfield $S$ coupled to $W_{\alpha} W^{\alpha}$, with $W_{\alpha}$ the chiral superfield strength, was analyzed in [12] and finite energy cosmic string solutions were constructed. Also, the impact of axions on dynamics of a $d=3+1$ Yang-Mills theory supporting non-Abelian strings has been analyzed in [16].

It is the purpose of this work to consider similar issues in $d=2+1$ space-time dimensions for which a rich variety of flux tube solutions exists already when the axion field is absent. Indeed, at high temperatures, a relativistic four dimensional quantum field theory becomes effectively three dimensional. Finite temperature calculations are carried out as usual by compactifying the (Euclidean) time variable in the range $0 \leq \tau \leq \beta=1 / T$. In the $T \rightarrow \infty$ limit, the time integral drops out and one remains with an action in $d=3$ Euclidean space. Now, the explicit break-

\footnotetext{
*Associated with CONICET.

${ }^{\dagger}$ Associated with CICBA.
}

ing in parity and $C P$ invariance induces a Chern-Simons term in the gauge-field effective action. It emerges, for example, in the presence of a nonzero fermionic number density. In this context the Chern-Simons coefficient is temperature dependent and proportional to $\mu$, the chemical potential of the fermionic U(1) charge [17].

When gauge fields with dynamics governed by a ChernSimons action are coupled to charged scalars with an appropriate sixth order symmetry breaking potential, the model admits BPS equations with vortexlike solutions carrying both magnetic flux and electric charge $[18,19]$ (see Ref. [20] for supergravity extension). It should be stressed that in the absence of the Chern-Simons term, electrically charged vortices with finite energy (per unit length) do not exist [21]. Hence, the model we are interested in, could show novel aspects of charged string like configurations when an axion is present, in particular with respect to their application to cosmological problems.

The paper is organized as follows: we start by considering, in Sec. II a purely bosonic model with couplings and potentials chosen so as to have a gauge invariant action leading to nontrivial Bogomolny equations. These equations are the natural extension, when an axion field is present, of those found in $[18,19]$. We present in Sec. III the supersymmetric extension of the model and we establish the connection between supersymmetry and BPS equations when the axion field is included. Numerical solutions of such first-order equations are presented in Sec. IV, where we discuss, in particular, novelties in the vortex solutions resulting from the presence of the axion field. A summary and discussion of our results are presented in Sec. V.

\section{AXION COUPLED TO A CHERN-SIMONS- HIGGS SYSTEM}

The coupling of an axion to a gauge field with dynamics governed by a Chern-Simons action poses some problems $[22,23]$. To discuss how can they be overcome, let us consider the following $(2+1)$-dimensional bosonic action, 


$$
\begin{aligned}
\mathcal{S}= & \int d^{3} x\left\{\frac{\kappa}{8 \delta} f(s) \operatorname{Im} D_{\mu} S \tilde{F}^{\mu}+\left|D_{\mu} \phi\right|^{2}\right. \\
& \left.+K^{\prime \prime}(s)\left|D_{\mu} S\right|^{2}-W(\phi, S)\right\},
\end{aligned}
$$

where $\phi$ and $S=s+i a$ are complex fields, $A_{\mu}$ is a $\mathrm{U}(1)$ gauge field, and $\tilde{F}^{\mu}$ is defined as

$$
\tilde{F}^{\mu} \equiv \epsilon^{\mu \nu \sigma} \partial_{\nu} A_{\sigma},
$$

$W\left(\phi, \phi^{*}, S, S^{*}\right)$ is a potential term, $f$ and $K$ are arbitrary functions of $s$, the real part of $S$, primes stand for $\partial / \partial s, \kappa$ is a constant and $\delta$ is a dimensionless parameter (which in the 4 dimensional case is related to the Fayet-Iliopoulos term and the Planck mass). Finally, $D_{\mu}$ is the covariant derivative acting on the fields $\phi$ and $S$ according to

$$
D_{\mu} \phi=\partial_{\mu} \phi-i e A_{\mu} \phi, \quad D_{\mu} S=\partial_{\mu} S+2 i \delta A_{\mu} \text {. }
$$

As done in [12] for the $3+1$ model, we shall identify $a$ in (1) with the axion field and $s$ with a dilaton field. Note that because of the definition of the axion covariant derivative, the Chern-Simons term appears in action (1) multiplied by the factor $f(s)$,

$$
\mathcal{S}_{\mathrm{CS}}[A, s]=\frac{\kappa}{4} \int d^{3} x f(s) \epsilon^{\mu \nu \sigma} A_{\mu} \partial_{\nu} A_{\sigma} .
$$

The action (1) is invariant under the local transformation

$$
\begin{gathered}
S \rightarrow S-2 i \delta \Lambda(x), \quad S^{*} \rightarrow S^{*}+2 i \delta \Lambda(x), \\
\phi \rightarrow e^{i e \Lambda(x)} \phi, \quad \phi^{*} \rightarrow \phi^{*} e^{-i e \Lambda(x),} \\
A_{\mu} \rightarrow A_{\mu}+\partial_{\mu} \Lambda(x) .
\end{gathered}
$$

The time component of the gauge-field equation of motion is the Chern-Simons version of the Gauss law and can be used to solve for $A_{0}$ giving

$$
A_{0}=\frac{\kappa \mathcal{B}}{2\left(e^{2}|\phi|^{2}+4 \delta^{2} K^{\prime \prime}\right)},
$$

where

$$
\mathcal{B} \equiv f(s) F_{x y}-\epsilon^{i j}\left(A_{i}+\frac{1}{2 \delta} \partial_{i} a\right) \partial_{j} f(s)
$$

The energy can be found from the energy-momentum tensor obtained by varying the action with respect to the metric,

$$
\delta S=\frac{1}{2} \int d^{3} x \sqrt{g} T^{\mu \nu} \delta g_{\mu \nu}
$$

Integration of the time-time component $T^{00}$ gives

$$
\begin{aligned}
\mathcal{E}= & \int d^{2} x\left\{\left|D_{i} \phi\right|^{2}+K^{\prime \prime}\left|D_{i} S\right|^{2}+W(\phi, S)\right. \\
& \left.+\frac{\kappa^{2} \mathcal{B}^{2}}{4\left(e^{2}|\phi|^{2}+4 \delta^{2} K^{\prime \prime}\right)}\right\} .
\end{aligned}
$$

After some work, this expression can be written in the form

$$
\begin{aligned}
\mathcal{E}= & \int d^{2} x\left(\left|D_{x} \phi \pm i D_{y} \phi\right|^{2}+K^{\prime \prime}\left|D_{x} S \pm i D_{y} S\right|^{2}+\frac{\kappa^{2}}{4\left(e^{2}|\phi|^{2}+4 \delta^{2} K^{\prime \prime}\right)}\left[\mathcal{B} \pm \frac{\left(e^{2}|\phi|^{2}+4 \delta^{2} K^{\prime \prime}\right)}{\kappa^{2} f(s) / 2}\left(e\left(|\phi|^{2}-\left|\phi_{0}\right|^{2}\right)-4 \delta K^{\prime}\right)\right]^{2}\right. \\
& +W-\frac{1}{(\kappa f(s))^{2}}\left(e^{2}|\phi|^{2}+4 \delta^{2} K^{\prime \prime}\right)\left(e\left(|\phi|^{2}-\left|\phi_{0}\right|^{2}\right)-4 \delta K^{\prime}\right)^{2} \pm\left(e\left(|\phi|^{2}-\left|\phi_{0}\right|^{2}\right)-4 \delta K^{\prime}\right) \epsilon^{i j}\left[A_{i}+\frac{1}{2 \delta} \partial_{i} a\right] \\
& \left.\times \partial_{j} \log f(s) \pm e\left|\phi_{0}\right|^{2} F_{x y}\right) .
\end{aligned}
$$

We thus see that the first three terms in Eq. (10) have been accommodated as perfect squares. This, together with an appropriate choice of the potential $W$ so as to cancel the fourth and fifth terms, would lead to a Bogomolny bound for the energy given by the magnetic flux $\Phi$ appearing in the last term,

$$
\Phi=\int d^{2} x F_{x y}
$$

or

$$
\Phi=2 \pi n
$$

for gauge fields with topological number $n \in \mathbb{Z}$. There is however the sixth term in (10) with no definite sign preventing the obtention of a bound. Only if we put $f(s)=1$, which corresponds to a normal Chern-Simons action for the gauge field [see Eq. (4)], this term vanishes. In that case one does have a bound,

$$
\mathcal{E} \geq \pm e\left|\phi_{0}\right|^{2} \Phi=2 \pi e\left|\phi_{0}\right|^{2}|n|,
$$

whenever the potential is chosen as

$$
W=\frac{1}{\kappa^{2}}\left(e^{2}|\phi|^{2}+4 \delta^{2} K^{\prime \prime}\right)\left(e\left(|\phi|^{2}-\left|\phi_{0}\right|^{2}\right)-4 \delta K^{\prime}\right)^{2} .
$$

The bound is saturated by fields obeying the self-duality equations

$$
\begin{aligned}
& D_{x} \phi=\mp i D_{y} \phi, \quad D_{x} S=\mp i D_{y} S, \\
& \kappa^{2} F_{x y}=\mp 2\left(e^{2}|\phi|^{2}+4 \delta^{2} K^{\prime \prime}\right)\left(e\left(|\phi|^{2}-\left|\phi_{0}\right|^{2}\right)-4 \delta K^{\prime}\right),
\end{aligned}
$$


where the upper (lower) sign corresponds to positive (negative) values of $\Phi$.

As it is well known, the presence of a Chern-Simons term forces a relation between magnetic flux and electric charge [24]. This makes the Chern-Simons vortices both magnetically and electrically charged. To see this phenomenon in the present case let us write the gauge-field equation of motion, for the case $f(s)=1$ in the form

$$
\frac{\kappa}{2} \varepsilon^{\mu \alpha \beta} F_{\alpha \beta}=J^{\mu}
$$

with $J^{\mu}=(\rho, \vec{J})$ the conserved matter current and $\rho$ the electric charge density,

$$
\begin{aligned}
J^{\mu}= & -2\left(e^{2}|\phi|^{2}+4 K^{\prime \prime} \delta^{2}\right) A^{\mu}-i e\left(\phi \partial^{\mu} \phi^{*}+\phi^{*} \partial^{\mu} \phi\right) \\
& -4 \delta \partial^{\mu} a .
\end{aligned}
$$

We then see that Eq. (6) can be rewritten in the form

$$
\rho=-\kappa \mathcal{B}
$$

so that the usual relation between electric charge $Q=$ $\int \rho d^{2} x$ and magnetic flux in Chern-Simons theories holds,

$$
Q=-\kappa \Phi .
$$

Note that both the Higgs scalar and the axion contribute to the electric charge.

\section{SUPERSYMMETRIC EXTENSION}

The SUSY extension of the Chern-Simons-Higgs system with a sixth order symmetry breaking potential was analyzed in [25]. Let us study now the case in which the axion field is also present.

Consider the following $d=3$ action, written in terms of superfields as

$$
\begin{aligned}
\mathcal{S}_{\text {SUSY }}= & -\frac{1}{2} \int d^{3} x d^{2} \theta\left(\frac{\kappa}{4 \delta} F\left(\Sigma+\Sigma^{\dagger}\right) \operatorname{Im}\left(\tilde{\nabla}_{a} \Sigma\right) W^{a}\right. \\
& +H\left(\Phi^{\dagger}, \Phi\right)\left(\nabla^{a} \Phi\right)^{\dagger} \nabla_{a} \Phi+K_{s \bar{s}}\left(\Sigma, \Sigma^{\dagger}\right)\left|\tilde{\nabla}_{a} \Sigma\right|^{2} \\
& \left.+2 V\left(\Phi^{\dagger}, \Phi, \Sigma, \Sigma^{\dagger}\right)\right) .
\end{aligned}
$$

The index $a$ takes the values 1,2 and $\Gamma_{a}$, $\Phi$, and $\Sigma$ are spinor, complex scalar and axionic superfields. Also

$$
\begin{gathered}
\nabla_{a} \Phi=\left(D_{a}-i e \Gamma_{a}\right) \Phi, \quad \tilde{\nabla}_{a} \Sigma=D_{a} \Sigma+2 i \delta \Gamma_{a}, \\
D_{a}=\frac{\delta}{\delta \theta^{a}}+i\left(\gamma^{\mu}\right)_{a b} \theta^{b} \partial_{\mu},
\end{gathered}
$$

where $\mu=0,1,2$.

The lowest component of $\Gamma^{a}, \Phi$, and $\Sigma$ are, respectively, the gauge field, the Higgs field and $S=s+i a$ with $a$ the axion field. Concerning $F, H, K$, and $V$, they are functionals of superfields to be fixed later. Subindices in these functionals indicate derivatives, thus $K_{s \bar{s}}=\partial_{S} \partial_{S^{*}} K=$ $\left.\partial_{\Sigma} \partial_{\bar{\Sigma}} K\right|_{\theta=0}$ and so on. These functionals should be chosen so that the supersymmetric action (20) is invariant under the supergauge transformations,

$$
\Phi \rightarrow e^{i e \Lambda} \Phi, \quad \Gamma_{a} \rightarrow \Gamma_{a}-D_{a} \Lambda, \quad \Sigma \rightarrow \Sigma_{a}-2 \delta \Lambda
$$

for any real scalar superfield $\Lambda$. Written in the WessZumino gauge, i.e.,

$$
\left.\Gamma_{a}\right|_{\theta=0}=\left.D^{a} \Gamma_{a}\right|_{\theta=0}=0,
$$

the spinor superfield $\Gamma_{a}$ is given by

$$
\Gamma_{a}(x, \theta)=i \theta^{b}\left(\gamma^{\mu}\right)_{b a} A_{\mu}(x)-2 \theta^{2} \lambda_{a}(x),
$$

where $\lambda(x)$ is a Majorana spinor, the photino. Then, the spinor field strength, defined as

$$
W_{a}=\frac{1}{2} D^{b} D_{a} \Gamma_{b}
$$

takes, in terms of component fields, the form

$$
W_{a}(x, \theta)=\lambda_{a}(x)-\frac{1}{2} \theta^{b}\left(\gamma^{\mu} \gamma^{\nu}\right)_{b a} F_{\mu \nu}-i \theta^{2}\left(\gamma^{\mu}\right)_{a}^{b} \partial_{\mu} \lambda_{b}(x)
$$

and satisfies the Bianchi identity,

$$
D^{a} W_{a}=0 .
$$

The complex scalar superfield is defined as

$$
\Phi(x, \theta)=\phi(x)+\theta^{a} \psi_{a}(x)-\theta^{2} F(x),
$$

where $\phi$ stands for the Higgs complex scalar field, $\psi_{a}$ is a Dirac bispinor, the Higgsino, and $F$ is a complex auxiliary field. Finally, the superfield $\Sigma$ which contains the axion $S$ as its lowest component, $\Sigma=S+\theta^{\alpha} \chi_{\alpha}-\theta^{2} J$ can be reduced to a complex scalar field $\Theta$ by exponentiation,

$$
\Theta=e^{\Sigma} \text {. }
$$

The supersymmetric transformations (21) take $\Gamma^{a}$ out from the Wess-Zumino gauge. One can however implement a composition of SUSY and gauge transformations such that the Wess-Zumino gauge remains valid. To do that, the new SUSY transformation for scalar and spinorial superfields are, respectively,

$$
\begin{gathered}
\delta_{\eta}^{\mathrm{WZ}} \Gamma_{a}=i \eta^{b} Q_{b} \Gamma_{a}+D_{a} \tilde{K}, \\
\delta_{\eta}^{\mathrm{WZ}} \Phi=i \eta^{b} Q_{b} \Phi+i \tilde{K} \Phi, \quad \delta_{\eta}^{\mathrm{WZ}} \Sigma=i \eta^{b} Q_{b} \Sigma+i \tilde{K},
\end{gathered}
$$

where $Q_{a}=i \partial_{a}+\theta^{b}\left(\gamma^{\mu}\right)_{b a} \partial_{\mu}, \eta^{a}$ is a Majorana spinor and the real scalar superfield $\tilde{K}$ is defined as

$$
\tilde{K}=i \theta^{a}\left(\gamma^{\mu}\right)_{a b} \eta^{b} A_{\mu}+\theta^{2} \lambda^{a} \eta_{a} .
$$

Let us restrict the action (20) to the case in which

$$
F\left(\Sigma+\Sigma^{\dagger}\right)=H\left(\Phi^{\dagger}, \Phi\right)=1 .
$$

Then, written in components, the action takes the simple form

$$
\mathcal{S}_{\mathrm{SUSY}}=\mathcal{S}_{\mathrm{B}}+\mathcal{S}_{\mathrm{F}}
$$

with the pure bosonic action given by 


$$
\mathcal{S}_{\mathrm{B}}=\int d^{3} x\left\{\frac{\kappa}{4} \epsilon^{\mu \nu \sigma} A_{\mu} F_{\nu \sigma}+\left|D_{\mu} \phi\right|^{2}+R\left|\tilde{D}_{\mu} S\right|^{2}-\left|V_{\phi}\right|^{2}-\frac{1}{R}\left|V_{s}\right|^{2}\right\},
$$

while the fermionic one is given by

$$
\begin{aligned}
\mathcal{S}_{\mathrm{F}}= & \int d^{3} x\left\{i \bar{\psi} \gamma^{\mu} \stackrel{\leftrightarrow}{D}_{\mu} \psi+i R \bar{\chi} \gamma^{\mu} \stackrel{\leftrightarrow}{\partial}_{\mu} \chi-\frac{1}{2} R_{s \bar{s}}(\bar{\chi} \chi)^{2}+\frac{1}{2}\left[i \bar{\chi} \gamma^{\mu} \chi\left(R_{s} \tilde{D}_{\mu} S-R_{\bar{s}} \tilde{D}_{\mu} S^{*}\right)\right]+\bar{\psi} \psi\left(\frac{e^{2}}{\kappa}|\phi|^{2}-V_{\phi \bar{\phi}}\right)\right. \\
& +\bar{\chi} \chi\left(\frac{4 \delta^{2}}{\kappa} R^{2}-V_{s \bar{s}}\right)-\bar{\psi} \chi\left(\frac{2 \delta e}{\kappa} \phi R+V_{\bar{\phi} s}\right)-\bar{\psi}^{*} \chi^{*}\left(\frac{2 \delta e}{\kappa} \phi^{*} R+V_{\phi \bar{s}}\right)-\frac{\left|R_{s}\right|^{2}}{4 R}\left|\bar{\chi} \chi^{*}\right|^{2}+\frac{1}{2} \bar{\psi}^{*} \psi\left(-V_{\phi \phi}\right. \\
& \left.-\frac{e^{2}}{\kappa} \phi^{* 2}\right)+\frac{1}{2} \bar{\psi} \psi^{*}\left(-V_{\bar{\phi} \bar{\phi}}-\frac{e^{2}}{\kappa} \phi^{2}\right)+\frac{1}{2} \bar{\chi}^{*} \chi\left(\frac{R_{s}}{R} V_{s}-V_{s s}-\frac{4 \delta^{2}}{\kappa} R^{2}\right)+\frac{1}{2} \bar{\chi} \chi^{*}\left(\frac{R_{\bar{s}}}{R} V_{\bar{s}}-V_{\bar{s} \bar{s}}-\frac{4 \delta^{2}}{\kappa} R^{2}\right) \\
& +\bar{\psi}^{*} \chi\left(\frac{2 \delta e}{\kappa} \phi^{*} R-V_{\phi s}\right)+\bar{\psi} \chi^{*}\left(\frac{2 \delta e}{\kappa} \phi R-V_{\bar{\phi} \bar{s})\},}\right.
\end{aligned}
$$

where we have redefined $R \equiv K_{s \bar{s}}$. Here we have taken into account that

$$
I=\int d^{3} x d^{2} \theta\left(D^{a} \Sigma\right) W_{a}
$$

is a surface term which does not modify the equations of motion

$$
\begin{aligned}
I & =\int d^{3} x d^{2} \theta D^{a}\left(\Sigma W_{a}\right)=\left.\int d^{3} x D^{2} D^{a}\left(\Sigma W_{a}\right)\right|_{\theta=0} \\
& =\left.i \int d^{3} x \partial_{b}^{a} D^{b}\left(\Sigma W_{a}\right)\right|_{\theta=0},
\end{aligned}
$$

and can hence be neglected.

In order to extend the $N=1$ supersymmetry to an $N=$ 2 supersymmetry, we shall follow [25] and allow the transformation parameter $\eta$ to be complex, i.e., $\eta^{*} \neq \eta$. Now, this is the same as making an $N=1$ SUSY transformation followed by a $\mathrm{U}(1)$ fermion phase rotation. Thus, the new transformation for fermions will be $\psi_{a} \rightarrow e^{i \alpha} \psi_{a}$ and $\psi_{a}^{*} \rightarrow$ $e^{-i \alpha} \psi_{a}^{*}$ and the same is valid for $\chi_{a}$ and $\chi_{a}^{*}$. The new SUSY transformations act then as rotations on the fermions and one can then see that the only terms which do not respect the extended SUSY invariance are those on the last three lines in (26). Hence, in order to get an $N=2$ supersymmetric model we need

$$
\begin{aligned}
& -V_{\phi \phi}-\frac{e^{2}}{\kappa} \phi^{* 2}=0, \quad \frac{2 \delta e}{\kappa} \phi^{*} R-V_{\phi s}=0, \\
& \frac{R_{s}}{R} V_{s}-V_{s s}-\frac{4 \delta^{2}}{\kappa} R^{2}=0, \quad \frac{2 \delta e}{\kappa} \phi R-V_{\bar{\phi} \bar{s}}=0,
\end{aligned}
$$

where $V=V(u, v)$, with $u=\phi^{*} \phi$ and $v=S+S^{*}$. These equations imply that

$$
\begin{gathered}
V_{u}=-\frac{e}{\kappa}(e h(u)-2 \delta r(v)), \\
V_{v}=-\frac{2 \delta}{\kappa}(2 \delta r(v)-e h(u)) R(v),
\end{gathered}
$$

where

$$
\frac{d}{d v} r(v)=R(v), \quad \frac{d}{d u} h(u)=1 .
$$

We obviously have $h=u-u_{0}=|\phi|^{2}-\left|\phi_{0}\right|^{2}$, and since $S+S^{*}=2 s$, then $R=K^{\prime \prime}$ and $r=2 K^{\prime}$ where primes stand for derivatives with respect to $s$. From (25) the potential is

$$
\begin{aligned}
W & =\left|V_{\phi}\right|^{2}+\frac{1}{R}\left|V_{s}\right|^{2} \\
& =\frac{1}{\kappa^{2}}\left(e^{2}|\phi|^{2}+4 \delta^{2} K^{\prime \prime}\right)\left[e\left(|\phi|^{2}-\left|\phi_{0}\right|^{2}\right)-4 \delta K^{\prime}\right]^{2},
\end{aligned}
$$

which is exactly what we obtained in (14).

In order to get the Bogomolny bound and the self-dual equations one can analyze the supercharge algebra as in [3]. Alternatively, one can directly consider the component field SUSY transformations $\left(\delta_{\eta} X=\eta^{a} \delta_{a} X\right)$,

$$
\begin{gathered}
\delta_{a} A_{\mu}=-i\left(\gamma_{\mu}\right)_{a}^{b} \lambda_{b}, \quad \delta_{a} \lambda_{b}=\frac{1}{2}\left(\gamma^{\mu} \gamma^{\nu}\right)_{a b} F_{\mu \nu} \\
\delta_{a} \phi=-\psi_{a}, \quad \delta_{a} S=-\chi_{a} \\
\delta_{a} \psi_{b}=\epsilon_{a b} F-i\left(\gamma^{\mu}\right)_{a b} D_{\mu} \phi \\
\delta_{a} \chi_{b}=\epsilon_{a b} J-i\left(\gamma^{\mu}\right)_{a b} \tilde{D}_{\mu} S \\
\delta_{a} F=i\left(\gamma^{\mu}\right)_{a}^{b} D_{\mu} \psi_{b}+2 \lambda_{a} \phi \\
\delta_{a} J=i\left(\gamma^{\mu}\right)_{a}^{b} \partial_{\mu} \chi_{b}+2 \lambda_{a}
\end{gathered}
$$

and their complex conjugated $\left(\delta X^{\dagger}=\eta^{* a} \delta_{a} X^{\dagger}\right)$ and reobtain Bogomolny Eqs. (15) just by putting all fermion fields to zero in (28) and then ask the SUSY transformations for $\psi_{a}$ and $\chi_{a}$ to vanish once the auxiliary fields have been written in terms of dynamical fields using their equations of motion. The first condition corresponds to a restriction to the bosonic sector, the second one implies that physical states are supersymmetry invariant.

\section{VORTEX-LIKE SOLUTIONS}

We present in this section some vortex solutions to the BPS equations of motion. We choose for the Khäler po- 
tential the form

$$
K=-M^{2} \log \left(S+S^{*}\right) .
$$

As in [12], we shall analyze separately two cases: first, we consider the case in which the vortex is supported by the Higgs field (" $\phi$-strings" solutions), in the sense that at infinity it behaves as in the ordinary Nielsen-Olesen vortex, with its winding number linked to the magnetic flux. Then, we shall consider the case in which the vortex is supported by the $s$ field, a solution that we shall call an "s-string." In this case it is the axion winding number which is related to the magnetic flux.

In the first case, in order to obtain $\phi$-string solutions we make the ansatz [12]

$$
\phi_{1}=f(r) e^{i n \theta}, \quad S=s(r)-2 i \delta m \theta, \quad A_{\theta}=n \frac{v(r)}{r},
$$

where $n$ is the topological charge of the Higgs and $m$ is the topological charge of the axion.

It is convenient to work with dimensionless variables by defining

$$
\begin{aligned}
& \tau=\alpha r, \quad \alpha=\frac{e^{2} \phi_{0}^{2}}{\kappa}, \quad x(\tau)=v(\tau / \alpha), \\
& y(\tau)=e \delta^{-1} s(\tau / \alpha), \quad z(\tau)=\phi_{0}^{-1} f(\tau / \alpha) .
\end{aligned}
$$

With this convention the equations read

$$
\begin{gathered}
x^{\prime}=-\frac{2 \tau}{|n|}\left(z^{2}+\frac{4 \beta}{y^{2}}\right)\left(z^{2}-1+\frac{4 \beta}{y}\right), \\
y^{\prime}=-\frac{2}{\tau}(|m|-|n| x), \\
z^{\prime}=\frac{z}{\tau}(1-x)|n|,
\end{gathered}
$$

where $\beta=M^{2} / \phi_{0}^{2}$. From the first two equations we can integrate $y(\tau)$ in terms of $z(\tau)$, obtaining

$$
y(\tau)=2(|n|-|m|) \log \tau-2 \log z(\tau)+k
$$

with $k$ an arbitrary integration constant. Thus, we end with a system of two first-order coupled differential equations for $x(\tau)$ and $z(\tau)$.

The boundary conditions for Eqs. (32) and (34) can be determined as follows. By Eq. (30), the function $x(\tau)$ must vanish at the origin, so Eq. (34) implies that $z(\tau)$ also vanishes at the origin as $\tau^{|n|}$. Indeed, Eqs. (32) and (35) imply that $x \sim \tau^{2}$ for $m=0$ and $x \sim \tau^{2} / \log (\tau)^{2}$ for $m \neq$ 0 .

For large $\tau$, the function $x$ tends to 1 , thus $z$ also tends to 1 unless $|n|=|m|$. In this last case, $z \rightarrow z_{0}$, where $z_{0}$ is the solution of the algebraic equation

$$
z_{0}^{2}+\frac{4 \beta}{k-2 \log z_{0}}=1
$$

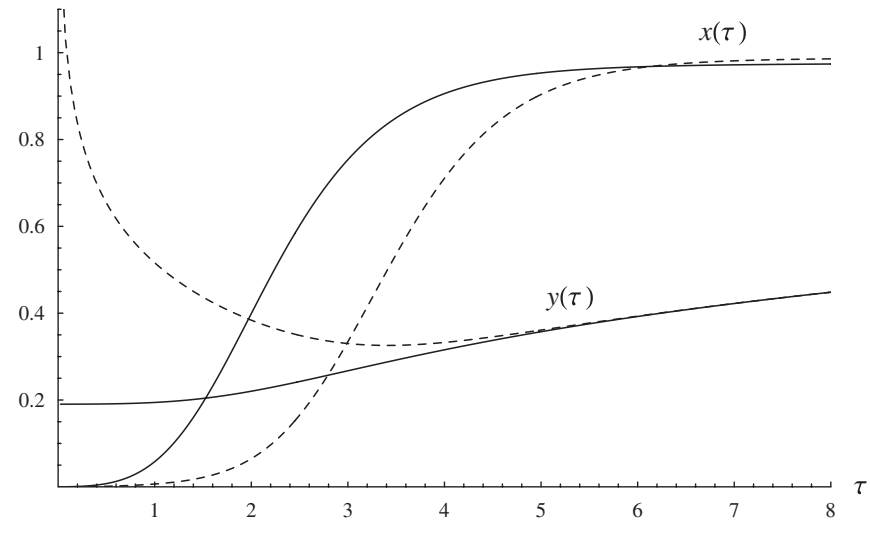

FIG. 1. Plot of the gauge field $x(\tau)$ and the dilaton $y(\tau)$ for $n=$ 1 and $m=0$ (solid line) and for $n=2$ and $m=1$ (dashed line). In both cases $\beta=1$ and $k=10$.

To solve the differential equations we employ a relaxation method for boundary values problem. Such a method determines the solution by starting with an initial guess and improving it iteratively. We present in Fig. 1 some solution profiles for the gauge field $x(\tau)$ and for the dilaton $y(\tau)$.

Already from ansatz (30) one can see that the magnetic flux and electric charge are quantized according to

$$
\Phi=\frac{2 \pi}{e} n, \quad Q=-\frac{2 \pi \kappa}{e} n
$$

There is an interesting property, typical of Chern-Simons vortices that also holds in our model: both the magnetic field and the (radial) electric field are concentrated in rings surrounding the zeroes of the Higgs field. We show this behavior in Fig. 2.

We also solved the BPS equations when the field $s$ tends asymptotically to a constant (" $s$-string" [12]). In this case the magnetic charge is equal to the topological charge of the axion field, so the appropriate ansatz is

$$
\phi=f(r) e^{i n \theta}, \quad S=s(r)-2 i \delta m \theta, \quad A_{\theta}=m \frac{v(r)}{r} \text {. }
$$

With this ansatz the BPS equations take the form

$$
\begin{gathered}
x^{\prime}=-\frac{2 \tau}{|m|}\left(z^{2}+\frac{4 \beta}{y^{2}}\right)\left(z^{2}-1+\frac{4 \beta}{y}\right), \\
y^{\prime}=-\frac{2}{\tau}|m|(1-x), \\
z^{\prime}=\frac{z}{\tau}(|n|-|m| x) .
\end{gathered}
$$

Again, we can integrate $y(\tau)$ in terms of $z(\tau)$, obtaining

$$
y(\tau)=2(|n|-|m|) \log \tau-2 \log z(\tau)+k .
$$

We see from this equation that for consistency, $z(\tau) \sim$ $\tau^{n-m}$ for $\tau \rightarrow \infty$ in contrast with what happens for the 


\section{B}
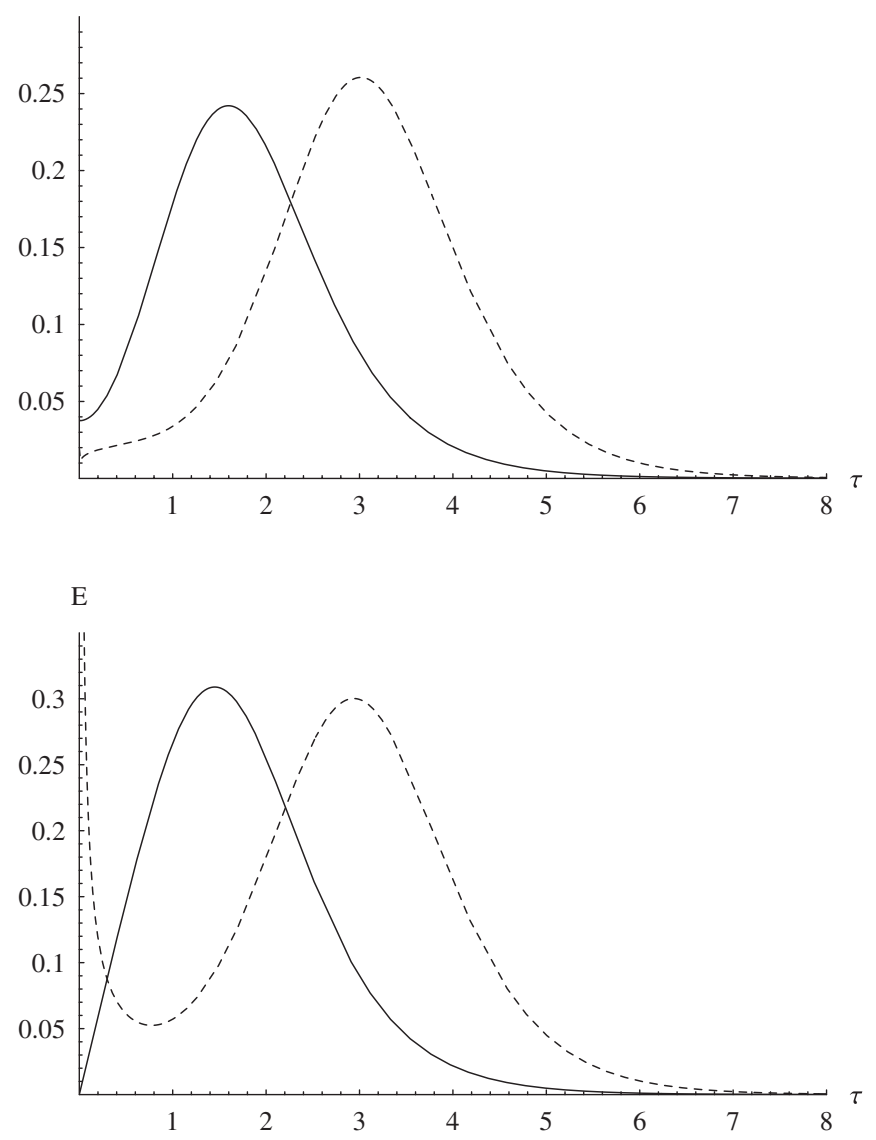

FIG. 2. Vortex magnetic and electric fields. The solid line corresponds to $n=1$ and $m=0$ and the dashed line to $n=2$, $m=1$. In both cases $\beta=1$ and $k=10$.

$\phi$-string. Concerning the gauge-field boundary condition, one has $\lim _{\tau \rightarrow \infty} x(\tau)=1$. We present in Fig. 3 some profile of the resulting $s$-string solutions.

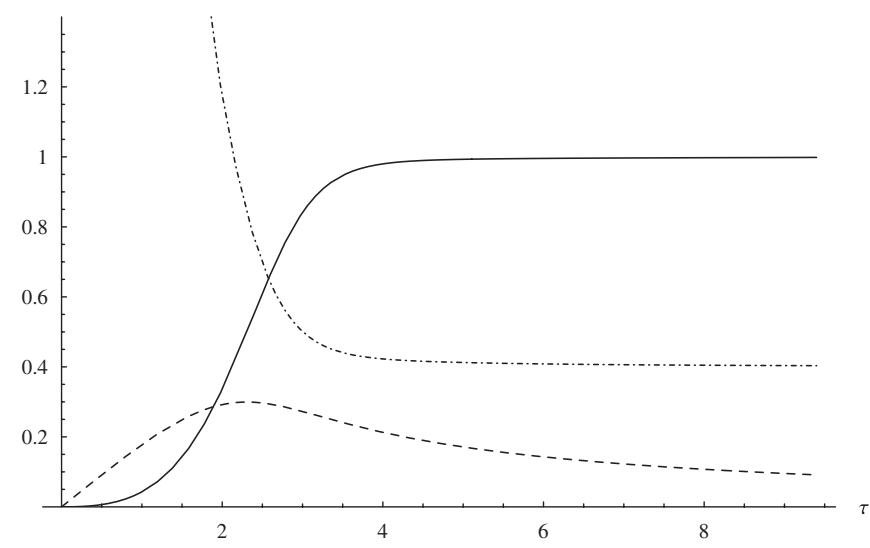

FIG. 3. Profile of an $s$-string solution. The solid line corresponds to the gauge field $x(\tau)$, the dot-dash line to the dilaton $y(\tau)$ and the dashed line to the Higgs field $z(\tau)$. For this solution $n=1, m=2, \beta=0.1$, and $k=0.1$.

\section{DISCUSSION}

We have been able to write first-order Bogomolny equations for the Chern-Simons-Higgs-axion system provided the gauge-field dynamics is governed by a normal ChernSimons action and the potential takes the form (14), with coupling constants satisfying the usual relation. As in the $3+1$ case discussed in [12], the first-order equation for the scalar $S$ containing the axion field has the same form as the ordinary Bogomolny equation for the Higgs field. The sixth order symmetry breaking potential (necessary to accommodate the energy as a sum of perfect squares plus a topological term) is modified due to the presence of the axion. As it always happens when matter is coupled to a Chern-Simons term, magnetic flux and electric charge turn to be proportional [see Eq. (19)]. Were we considering nonAbelian gauge fields, then the proportionality constant $\kappa$ should be quantized, implying in turn quantization of both electric charge and magnetic flux at the classical level.

The same constraints and results were found by constructing the SUSY extension of the purely bosonic model. In this case we have seen that the conditions on the potential and coupling constants arise when extending supersymmetry from $N=1$ to $N=2$ while the BPS equations can be inferred by asking physical states to be annihilated by SUSY generators.

The numerical solutions to the BPS equations presented in Sec. IV show that the axionless string solutions found in $[18,19]$ are not much modified by the axion which, however, contributes to the electric charge of the string configuration in a clear way. These explicit solutions could be of relevance in the context of cosmic strings and, due to the coupling to the axion and their electric charge, their dynamics could be very different from that usually considered. This, and the extension to the non-Abelian case (where both the magnetic flux and the electric charge are quantized) are issues that we hope to address in a future work.

Finally, a natural question to ask is wether the incorporation of a Maxwell term in addition to the Chern-Simons one, modify the soliton solutions. However is easy to see that these two terms dominate in opposite regimes, the Chern-Simons term in the IR and the Maxwell term in the UV. In particular, the Maxwell term decouples from the Chern-Simons-Higgs Lagrangian in the limit $e^{2} \rightarrow \infty$. Furthermore, analysis of numerical vortices in ChernSimons models with and without a Maxwell term show no significant differences between the two cases.

\section{ACKNOWLEDGMENTS}

The work of J. L.-S. was supported in part by Proyecto FONDECYT 3060 002. The work of F. A. S. was supported in part by Grant Nos. 6160 PIP-CONICET, CICBA, and X319 UNLP. J.L.-S. would like to thank La Plata University and USACH for hospitality while this work was been performed. 


\section{APPENDIX: CONVENTIONS}

We use the following representation of the $\gamma$ matrices,

$$
\left(\gamma^{0}\right)_{a}^{b}=\left(\sigma^{2}\right)_{a}^{b}, \quad\left(\gamma^{1}\right)_{a}^{b}=\left(i \sigma^{3}\right)_{a}^{b}, \quad\left(\gamma^{2}\right)_{a}^{b}=\left(i \sigma^{1}\right)_{a}^{b} .
$$

Thus,

$$
\gamma^{\mu} \gamma^{\nu}=g^{\mu \nu}-i \epsilon^{\mu \nu \sigma} \gamma_{\sigma}
$$

The "metric" in spinor space is given by

$$
C_{a b}=i\left(\sigma^{2}\right)_{a b}=\left(\begin{array}{cc}
0 & 1 \\
-1 & 0
\end{array}\right)=C^{a b},
$$

So

$$
C^{a b} C_{c d}=\delta_{[c}^{a} \delta_{d]}^{b}
$$

In particular,

$$
\psi^{a}=C^{a b} \psi_{b}, \quad \psi_{a}=\psi^{b} C_{b a}, \quad \theta^{2}=\frac{1}{2} \theta^{a} \theta_{a}=\theta_{2} \theta_{1} .
$$

We define the integration measure in the superspace

$$
\int d^{2} \theta \theta^{2}=-1
$$

Also, useful formulae are

$$
\begin{gathered}
\theta_{a} \theta_{b}=-C_{a b} \theta^{2}, \quad \theta^{a} \theta^{b}=-C^{a c} \theta^{2}, \\
\theta^{a} \theta_{b}=\delta_{b}^{a} \theta^{2}=-\theta_{b} \theta^{a}, \quad A^{[a} B^{b]}=-C^{a b} A^{c} B_{c}, \\
A_{[a} B_{b]}=-C_{a b} A^{c} B_{c},
\end{gathered}
$$

The $\gamma$ matrices are real and symmetric when lowering their indices, i.e.,

$$
\begin{aligned}
& \left(\gamma^{0}\right)_{a b}=\left(\gamma^{0}\right)_{a}^{c} C_{c b}=i I_{a b}\left(=i \delta_{a b}\right), \\
& \left(\gamma^{1}\right)_{a b}=\left(\gamma^{1}\right)_{a}^{c} C_{c b}=i\left(\sigma^{1}\right)_{a b}, \\
& \left(\gamma^{2}\right)_{a b}=\left(\gamma^{2}\right)_{a}^{c} C_{c b}=-i\left(\sigma^{3}\right)_{a b} .
\end{aligned}
$$

Then, any vectorial representation can be written in terms of the $\gamma$ matrices with spinorial indices, i.e.

$$
V_{a b}=\left(\gamma^{\mu}\right)_{a b} V_{\mu},
$$

where, $\gamma_{a b}$ are imaginary and symmetric $2 \times 2$ matrices. Also, we can define the space-time derivative in terms of spinorial indices,

$$
\partial_{a b}=\left(\gamma^{\mu}\right)_{a b} \partial_{\mu} .
$$

The spinorial derivative is define as

$$
\partial_{a}=\frac{\partial}{\partial \theta^{a}}, \quad \partial^{a}=-\frac{\partial}{\partial \theta_{a}},
$$

$$
\partial_{a b}=\frac{\partial}{\partial x^{a b}}, \quad \partial_{b}^{a}=-\frac{\partial}{\partial x_{a}^{b}} .
$$

Then, the supersymmetric covariant derivative can be written in terms of this notation as

$$
D_{a}=\partial_{a}+i \theta^{b} \partial_{b a}
$$

Note that the derivatives $\frac{\partial}{\partial \theta^{a}}$ and $\frac{\partial}{\partial x^{a b}}$ do not raise and lower indices in the same way as spinors,

$$
\begin{aligned}
& \partial_{a}=\left(\partial_{a} \theta_{b}\right) \frac{\partial}{\partial \theta_{b}}=C_{d b}\left(\partial_{a} \theta^{d}\right) \frac{\partial}{\partial \theta_{b}}=C_{a b} \frac{\partial}{\partial \theta_{b}}=C_{b a} \partial^{b}, \\
& \partial_{b a}=\frac{\partial x_{d}^{c}}{\partial x^{b a}} \frac{\partial}{\partial x_{d}^{c}}=C_{e d} \frac{\partial x^{c e}}{\partial x^{b a}} \frac{\partial}{\partial x_{d}^{c}}=C_{a d} \frac{\partial}{\partial x_{d}^{b}}=C_{d a} \partial_{b}^{d}
\end{aligned}
$$

causing the appearance of a minus sign in the definitions of $\partial^{a}$ and $\partial_{b}^{a}$ in (A3) and (A4).
[1] E. Witten, Phys. Lett. B 153, 243 (1985).

[2] J. Polchinski, hep-th/0412244.

[3] J. D. Edelstein, C. Núñez, and F. Schaposnik, Phys. Lett. B 329, 39 (1994).

[4] J. D. Edelstein, C. Núñez, and F. A. Schaposnik, Nucl. Phys. B458, 165 (1996).

[5] J.D. Edelstein, C. Núñez, and F. A. Schaposnik, Phys. Lett. B 375, 163 (1996).

[6] M. Shifman and A. Yung, Phys. Rev. D 70, 025013 (2004).

[7] G. Dvali, R. Kallosh, and A. Van Proeyen, J. High Energy Phys. 01 (2004) 035.

[8] P. Binetruy, G. Dvali, R. Kallosh, and A. Van Proeyen, Classical Quantum Gravity 21, 3137 (2004).

[9] A. Achucarro and J. Urrestilla, J. High Energy Phys. 08 (2004) 050.
[10] R. Jeannerot and M. Postma, J. High Energy Phys. 12 (2004) 043.

[11] S. S. Gubser, C.P. Herzog, and I. R. Klebanov, C.R. Physique 5, 1031 (2004).

[12] J. J. Blanco-Pillado, G. Dvali, and M. Redi, Phys. Rev. D 72, 105002 (2005).

[13] S. L. Parameswaran, G. Tasinato, and I. Zavala, Nucl. Phys. B737, 49 (2006).

[14] A. Achucarro, A. Celi, M. Esole, J. Van den Bergh, and A. Van Proeyen, J. High Energy Phys. 01 (2006) 102.

[15] R. Auzzi, M. Shifman, and A. Yung, hep-th/0511150.

[16] A. Gorsky, M. Shifman, and A. Yung, hep-th/0601131.

[17] A. N. Redlich and L. C. R. Wijewardhana, Phys. Rev. Lett. 54, 970 (1985).

[18] J. Hong, Y. Kim, and P. Y. Pac, Phys. Rev. Lett. 64, 2230 
(1990).

[19] R. Jackiw and E. J. Weinberg, Phys. Rev. Lett. 64, 2234 (1990).

[20] M. Abou-Zeid and H. Samtleben, Phys. Rev. D 65, 085016 (2002).

[21] B. Julia and A. Zee, Phys. Rev. D 11, 2227 (1975).

[22] M. Burgess, Phys. Rev. D 52, 1165 (1995).
[23] W. Garcia Fuertes and J. Mateos Guilarte, J. Math. Phys. (N.Y.) 38, 6214 (1997).

[24] S. Deser, R. Jackiw, and S. Templeton, Phys. Rev. Lett. 48, 975 (1982); S. Deser, R. Jackiw, and S. Templeton, Ann. Phys. (N.Y.) 140, 372 (1982).

[25] C. k. Lee, K. M. Lee, and E. J. Weinberg, Phys. Lett. B 243, 105 (1990). 